\title{
Separation of Madecassoside and Madecassic Acid Isomers by High Performance Liquid Chromatography Using $\beta$-Cyclodextrin as Mobile Phase Additive
}

\author{
Guiqing Kai, Jian Pan, Chuanxun Yuan, and Yuan Yuan \\ Engineering Research Center of Bio-process Ministry of Education, Hefei Cniversity of Technologn, Hefei, Anhwi 230069, China \\ E-mail: stroll46ahotmail.com \\ Received April 25, 2007
}

\begin{abstract}
An improved HPLC method for the separation of madecassoside isomers (madecassoside and asiaticoside-B) has been developed. The isomers can be separated with high resolution from extracts of Centella asiatica by HPLC using $\beta$-cyclodextrin as a mobile phase added on a $\mathrm{C}_{18}$ column. The result shows that the isomers can be separated with a mobile phase consisting of methanol:water $(50: 50 \mathrm{v} / \mathrm{v})$ with $+\mathrm{mmol} / \mathrm{L} \beta \mathrm{CD}$. To elucidate the mechanism of the separation of madecassoside and asiaticoside- $\mathrm{B}$, this paper studied the separation of their aglycon parts (madecassic acid and terminolic acid), another pair of isomers. The isomers can also be separated with high resolution with a mobile phase consisting of methanol:water $(65: 35, \mathrm{w} / \mathrm{v})$ with $+\mathrm{mmol} / \mathrm{L} \beta \mathrm{CD}$ and the $\mathrm{pH}$ of the mobile phase was adjusted to 4 . The paper also studied the separation of the two isomers by HPLC using $\alpha-\mathrm{CD}$ and Glucosyl- $\beta-\mathrm{CD}$ as a mobile phase additive in order to elucidate the mechanism of the separation process.
\end{abstract}

Key Words : Madecassoside, Madecassic acid, Isomer. HPLC. Cyclodextrin

\section{Introduction}

Centella asiatica (L.) Urb.. also known as gotu kola and Indian pennywort. is a tropical medicinal plant with a long history of therapeutic use e.g. dermal disorders. venous insufficiency and microangiopathy. Research has shown that Centella increased collagen synthesis in vitro and extracellular matrix accumulation in vivo. It enhanced tensile strength in wound tissue, and facilitated the wound healing process. ${ }^{\text {l-t }}$ There are many constituents in Centella. such as $\beta$-sitosterol. hexacosanol octanoate kaempferol quercetin. daucosterol vanillic acid. succinic acid asiatic acid madecassic acid. teminolic acid asiaticoside asiaticodiglycoside. madecassoside. asiaticoside-B. etc. Research has shown that there are many pairs of isomers in Centella, which are hardly separated by traditional methods. ${ }^{56}$ Madecassoside and asiaticoside-B and their aglycon parts (madecassic acid and terminolic acid) are two pairs of isomers. Their structures are shown in Figure 1.

The most common methods for separation of active constituents in Centella are liquid chromatography. ${ }^{7-\bar{y}}$ The separation of asiaticoside. madecassoside. madecassic acid and asiatic acid was studied in most literatures. But the study about separation of the pairs of isomeric compounds (madecassoside and asiaticoside-B or madecassic acid and terminolic acid) was rather sparse. There is a difference in molecular structure between madecassoside and asiaticoside$B$. or madecassic acid and terminolic acid. The difference of these compounds with $\beta$-CD may result in their different retention times. In fact cyclodextrin has already been used

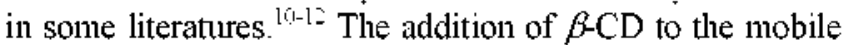
phase was therefore investigated to improve the separation. Because $\alpha$-CD has smaller cavity structure than $\beta-C D$ and Glu- $\beta$-CD has better hydrophilic property than $\beta$ CD. To

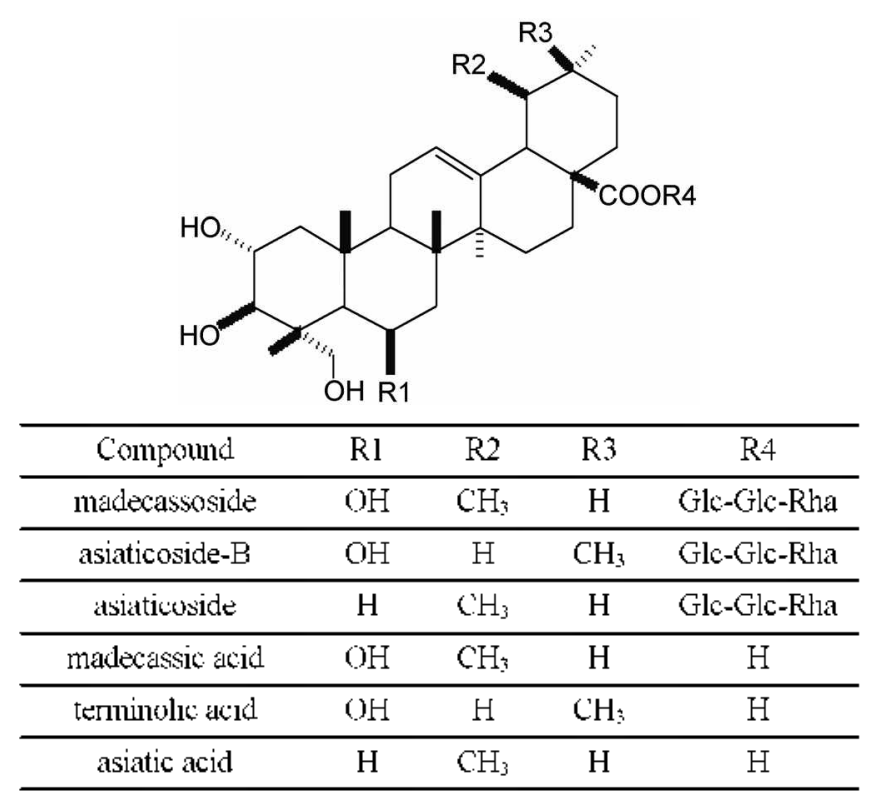

Figure 1. The structures of madecassoside, asiaticoside-B, asiaticoside and their aglycon parts (madecassic acid, teminolic acid and asiatic acid $\mathrm{j}$

discuss the mechanism of the separation process. the paper also separated the two isomers by HPLC using $\alpha$-CD and Glu- $\beta$-CD as a mobile phase additive.

This paper describes that the isomers (madecassoside and asiaticoside-B or madecassic acid and terminolic acid) can be separated from extracts of Centella by HPLC using $\beta$-CD or Glu- $\beta$-CD as a mobile phase additive. The isomers can be separated with high resolution in one step from extracts of Centella. But $\beta$-CD is cheaper than Glu- $\beta$-CD and harmless to the health. The method using $\beta \mathrm{CD}$ as a mobile phase additive by HPLC is cheap. simple and harmless in the 
separation and purification of the isomeric compounds of triterpenes or triterpenic genins in Centella.

\section{Experimental Section}

Reagents and equipments. Reference standard of madecassic acid was purchased from SIGMA. USA. Reference standard of madecassoside was purchased from National Institute for the Control of Phannaceutical and Biological Product of China (Beijing. China). The extract powder of $70 \%$ total Centella triterpenes and $50 \%$ Centella triterpenic genins were bought from Guangxi Changzhou Natural products Development Co. Ltd (Guangxi. China). $\beta$-Cyclodextrin $(\beta C D)$ was obtained from the Fine Chemicals Factory. Nankai University (Tianjin, China). $\alpha$-Cyclodextrin $(\alpha-C D)$ was purchased from SIGMA. USA. Glucosyl- $\beta$ Cyclodextrin (Glu- $\beta \mathrm{CD}$ ) bought from Shandong Xinda Group Co. Ltd.

The Waters HPLC system consists of a high-pressure constant flow puntp (model 515), 996 Photodiode Array Detector and a manual injection with a $10-\mu \mathrm{L}$ fixed loop. The analytical colunn was an Agilent Zorbax SB-C 18 ( 150 $\mathrm{mm} \times 4.6 \mathrm{~mm}$ i.d. $5 \mu \mathrm{mm}) .0 .4 \mathrm{~mL} / \mathrm{min}, 204 \mathrm{~nm}, 25^{\circ} \mathrm{C}$. LC. MS analysis was carried out using an Agilent 1100 LC-MSD system.

Sample preparation. The $70 \%$ total Centella triterpenes was accurately weighed $(40 \mathrm{mg})$, dissolved in methanol made up to $10 \mathrm{~mL}$. The solution was filtered through a 0.45 $\mu \mathrm{m}$ filter before use. The sample preparation for $50 \%$ total triterpenic genins. reference standard of madecassoside and reference standard of madecassic acid were processed by the same way.

Mobile phases. The mobile phase for separation of madecassoside and asiaticoside- $\mathrm{B}$ consists of methanol: water $(50.50, \mathrm{v} / \mathrm{v})$ with $4 \mathrm{mmol} / \mathrm{L} \beta-\mathrm{CD}$ or $4 \mathrm{mmol} / \mathrm{L}$ Glu- $\beta-\mathrm{CD}$. The mobile phase for separation of madecassic acid and terminolic acid consists of methanol: water $(65: 35, \mathrm{v} / \mathrm{v})$ with $4 \mathrm{mmol} / \mathrm{L} \beta-\mathrm{CD}$ or $4 \mathrm{mmol} / \mathrm{L}$ Glu- $\beta$ CD. The funal $\mathrm{pH}$ of the mobile phase was adjusted to 4 . The mobile phases were filtered through $0.45 \mu \mathrm{m}$ filters before use.

Collecting madecassoside, asiaticoside-B, madecassic acid and terminolic acid. The HPLC with $\beta$ CDD as a mobile phase additive was used repetitively to collect sufficient amounts of madecassoside. asiaticoside-B. madecassic acid and terminolic acid for the analysis of configuration. The solutes were evaporated to dry'ness. get rid of $\beta \mathrm{CD}$, dissolved in methanol and filtered through $0.45 \mu \mathrm{m}$ filters.

\section{Results and Discussion}

Application of $\beta$-CD. The result of separation of the madecassoside isomers by HPLC using $\beta \mathrm{CD}$ as an addition of mobile phase is shown in Figure 2. The nobile phases of different systems and proportions were compared. Clear baseline resolution was observed in B while only single chromatographic peak (I) was observed in A. That is to say that the resolution between the madecassoside isomeris
(A)

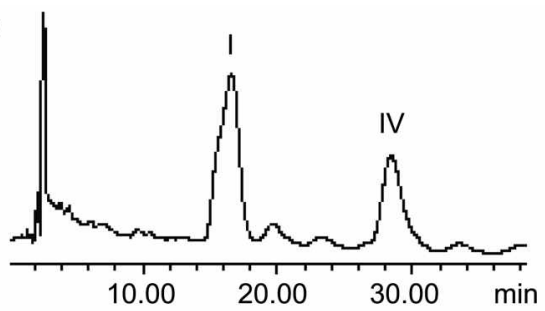

(B)

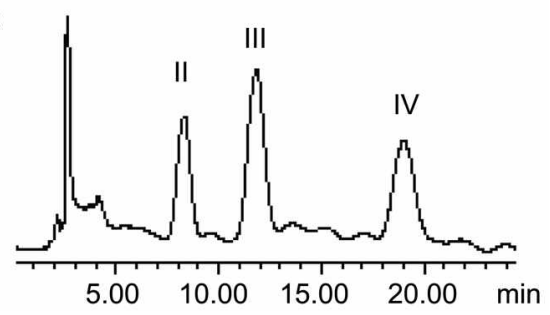

Figure 2. Chromatograms of $70 \%$ total Centella triterpenes Mobile phase consisting of: (A) methanol water solution $(50: 50, \mathrm{v} /$ $\mathrm{vi}$ (B) methanol water solution $(50: 50, \mathrm{v} / \mathrm{v})$ with adding $4 \mathrm{mmol} / \mathrm{L}$ $\beta$ CD. (II is asiaticoside-B, III is madecassoside and IV is asiaticoside).

could be increased obviously by adding $\beta \mathrm{CD}$ into the mobile phases. The retention times of isomers were both brought fonvard comparing to the mobile phase without $\beta$ $C D$. The area of peak was equal to the area plus the area approximately.

$\beta \mathrm{CD}$ is composed of seven cyclic-arranged $\alpha-1,4$-linked glucose units. The outside of the cyclic molecule is hydrophilic and the central cavity is hydrophobic. Generally, using $\beta \mathrm{CD}$ as additional mobile phase, the polarity of mobile phase will enhance which makes the retention times delay. However. the opposite result was observed in B. The reasonable explanation might be that the outside of the cyclic molecule is hydrophilic and madecassoside and asiaticoside-B include hydrophilic glucoses. With interaction between the isomers and $\beta \mathrm{CD}$. madecassoside and asiaticoside- $\mathrm{B}$ were easier to fluid out. which brought the retention times forward.

The molecular weights of the collected peak II and III have been determined from LC.MSD [m/z: $\left.997(\mathrm{M}+\mathrm{Na})^{-}\right]$. which are the same molecular weight (974) as madecassoside and asiaticoside-B. From the reported studies." the isomers can only become single peak in common HPLC because of their similar characters and the area of peak I was

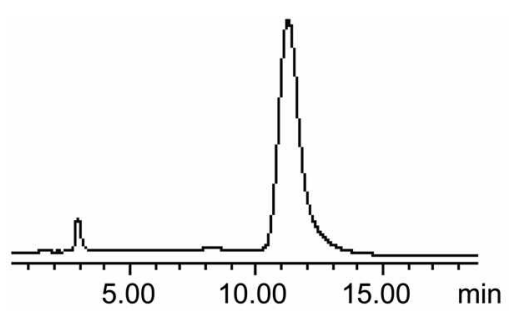

Figure 3. Chromatograms of reference standard of madecassoside. Mobile phase consisting of: methanol water solution $(50: 50, v / v)$ with $4 \mathrm{mmol} / \mathrm{L} \beta \mathrm{CD}$ added. 
(A)

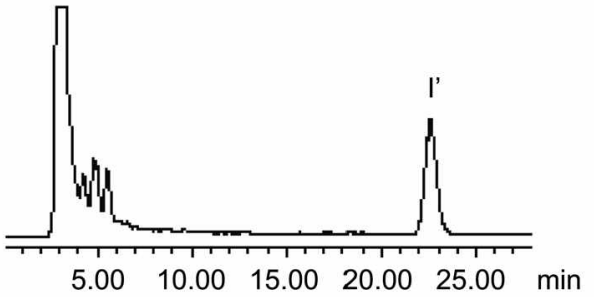

(B)

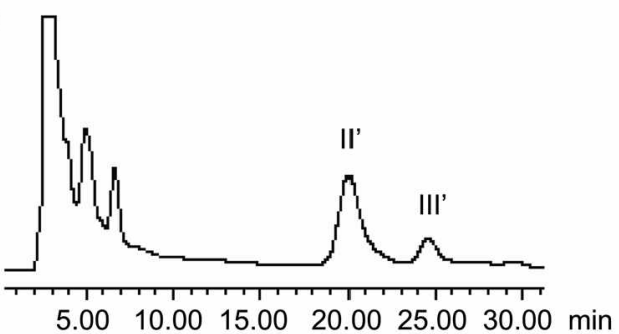

Figure 4. Chromatograms of $50 \%$ Centella triterpenic genins. Mobile phase consisting of: (A) methanol water solution (65:35, $\mathrm{v} / \mathrm{v}, \mathrm{pH}=4)$, (B) methanol water solution $(65: 35, \mathrm{v} / \mathrm{v}, \mathrm{pH}=4)$ with adding $4 \mathrm{mmol} / \mathrm{L} \beta \mathrm{CD}$. (II' is terminolic acid and III is madecassic acid).

equal to the area II plus the area III approximately. So that peak II and III are the isomers (madecassoside and asiaticoside-B). Moreover. peak III is madecassoside by reference standard of madecassoside (Fig. 3). Peak II may be asiaticoside-B.

To test whether madecassoside and asiaticoside-B's aglycon parts (madecassic acid and terminolic acid) played a great job in separation of the isomers, clromatography was used for separation of madecassic acid and terminolic acid. The mobile phases of different systems and proportions were compared in Figure 4 . That shows the resolution between the madecassic acid isomeris could also be improved by adding $\beta-\mathrm{CD}$ into the mobile phases

The molecular weights of the collections of chromatographic peak II' and III' have been determined from LCMSD [m/z: $\left.527(\mathrm{M}+\mathrm{Na})^{-}\right]$, which are the same molecular weight (504) as madecassic acid and terminolic acid. So. peak II' and III' are the isomers (teminolic acid and madecassic acid). Moreover. peak III' is madecassic acid by reference standard of madecassic acid (Fig. 5). Peak II' may be terminolic acid.

The outside of the $\beta$-CD's cyclic molecule is hydrophilic and the central cavity is hydrophobic. Madecassoside and asiaticoside-B's aglycon parts are hydrophobic. It is known that the hydrophobic inner core of $\beta$-CD has the ability to absorb hydrophobic solutes, it is reasonable to assume that the absorption property of $\beta$-CD and the aglycon parts are based on this property. However, the molecular structure of madecassic acid belongs to ursane category while teminolic acid' molecular structure belongs to oleanane category. The different complexation results from different spaces block lead to different chromatography behaviors. which result in different retention times of madecassic acid and teminolic acid.

The madecassoside isomers can be separated with high

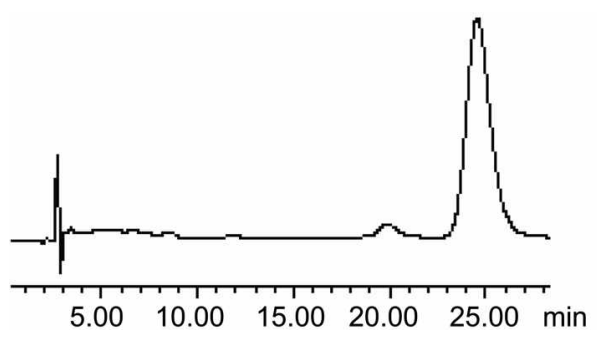

Figure 5. Chromatograms of reference standard of madecassic acid. Mobile phase consisting of: methanol water solution $655: 35$, $\mathrm{v} / \mathrm{v}, \mathrm{pH}=4$ ) with $4 \mathrm{mmol} / \mathrm{L} \beta \mathrm{CD}$ added.

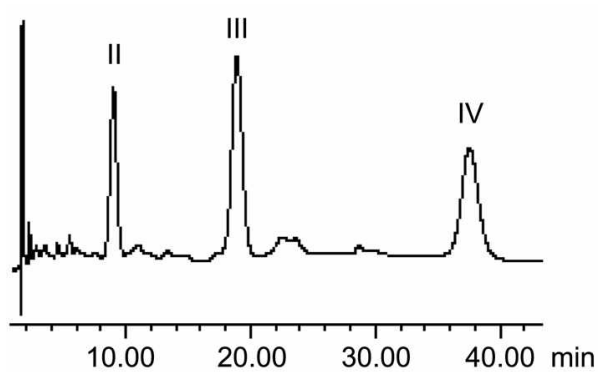

Figure 6. Chromatograms of $70 \%$ total Centella triterpenes. Mobile phase consisting of: methanol water solution $(50: 50, \mathrm{v} / \mathrm{v})$ with adding $4 \mathrm{mmol} / \mathrm{L}$ Glu- $/$ CD. (II is asiaticoside- $\mathrm{B}$, III is madecassoside and IV is asiaticoside)

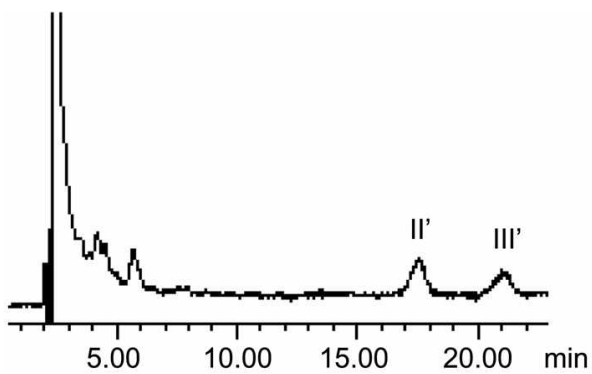

Figure 7. Chromatograms of $50 \%$ Centella triterpenic genins Mobile phase consisting of: methanol water solution $(65: 35, \mathrm{v} / \mathrm{v}$, $\mathrm{pH}=4$ ) with adding $4 \mathrm{mmol} / \mathrm{L}$ Glu- $\beta$ C.C. (II' is teminolic acid and III' is madecassic acid).

resolution by the adding $\beta \mathrm{CD}$. while the madecassic acid isomers can also be separated by the adding $\beta \mathrm{CD}$. But the retention times of madecassoside isomers were both brought forward comparing to the mobile phase without $\beta \mathrm{CD}$, while the madecassic acid were not obvious. That is to say it may be the aglycon parts of madecassoside and asiaticoside-B (madecassic acid and teminolic acid) that plays a great role in the separation process.

Application of Glu- $\beta$-CD. The result of separation of the madecassoside and madecassic acid isomers by HPLC using Glu- $\beta$-CD as an addition of mobile phase is shown in Figure 6 and Figure 7. That shows the resolution between the madecassoside and madecassic acid isomers could be improved by adding Glu- $\beta$ CD into the mobile phases.

From the tablel. there is higher resolution in separation of the two isomers by HPLC using $\beta \mathrm{CD}$ as additional mobile phase than using Glu- $\beta$-CD. It is may be that Glu- $\beta$ CD 
Table 1. The contrast between different mobile phases

\begin{tabular}{|c|c|c|c|c|}
\hline \multirow{2}{*}{ Sample } & \multicolumn{2}{|c|}{ Mobile phase with adding 4 mmol/L $\beta$ CD } & \multicolumn{2}{|c|}{ Mobile phase with adding $4 \mathrm{mmol} / \mathrm{L}$ Glu- $\beta C \mathrm{CD}$} \\
\hline & Retention time & Resolution & Retention time & Resolution \\
\hline Asiaticoside-B & 8.319 & \multirow{2}{*}{2.04} & 11.096 & \multirow{2}{*}{4.55} \\
\hline Madecassoside & 11.843 & & 18.948 & \\
\hline Terninolic acid & 20.141 & \multirow{2}{*}{2.12} & 17.596 & \multirow{2}{*}{2.48} \\
\hline Madecassic acid & 24.542 & & 21.033 & \\
\hline
\end{tabular}

includes more hydrophilic glucoses which enhance the interaction between the isomers and cyclodextrins.

Application of $\alpha$-CD. The resolution between the madecassoside isomeris couldn't be increased by adding $\alpha-C D$ into the mobile phases. It is may be $\alpha$-CD has sntaller cavity structure than $\beta C D$. The isomers molecule couldn't be absorbed into the cavity of $\alpha-C D$. The interactional force between the isomers and $\alpha-C D$ is too weak to separate the isomers

\section{Conclusion}

The isomers (madecassoside and asiaticoside-B) can be separated with high resolution from extracts of Centella by HPLC using $\beta$ CD as an addition of mobile phase. Their aglycon parts (madecassic acid and terminolic acid) can be separated with high resolution, too. The paper has explained that it may be the aglycon parts of madecassoside and asiaticoside- $B$ that plays a great role in the separation process. Moreover. the two isomers can also be separated with higher resolution by HPLC with Glu- $\beta$-CD as a mobile phase additive. But $\alpha$-CD can t separate of the two isomers in the same condition. That is to say the hydrophilic group and the size of cavity structure of $C D$ play the leading role in the separation process. Furthermore, $\beta$-CD is harmless to the health and cheaper than Glu- $\beta$ CD. The method using $\beta$ CD as a mobile phase additive by HPLC is cheap. simple and harmless in the separation and purification of the isomers of triterpenes or triterpenic genins in Centella.

Acknowledgements. This work was supported by Grant 20136020 from the National Natural Science Foundation of China.

\section{References}

1. Qin. L. P.: Zhuang. W. G.: Zheng. H. C.: Zhang. L. World Notes: Phutomed 1997, 12(4). 154.

2. Zainol. M. K.: Abd-Hamid. A.: Yusof. S.: Muse. R. Food Chent. 2003. 81.575

3. Cheng. C. L.; Guo, J. S.: John, L.; Marcel, W. L. K. Life Sci. 2004. $7+.2237$.

4. Babu. T. D.: Uttan. G. K.: Padikiala. J. J Ethophamacol 1995. 48. 53 .

5. Matsuda. H.: Morikawa. T.: Ueda. H.: Yoshikawa. M. Chent Pham Bull. 2001, 49(10), 1368.

6. Sahu. N. P.: Roy. S. B. Plntochemisty 1989. $28(10), 2852$.

7. Inamdar. P. K.: Yeole. R. D.: Ghogare. A. B.: Souza. N. J. de $J$ of Chomatography A 1996, 742, 127 .

8. Schaneberg. B. T.: Mikell. J. R.: Bedir. E.: Khan. I. A. Phomazie 2003. $58(6) .381$.

9. Zhang. L. L.: Wang. H. S: Yao. Q. Q: Liu. Y. J.: Luan. Y: Wang. X. L. Chinese Traditional and Herbal Dngs 2005. 36(12). 1761.

10. Kim. H.: Jeong, K; Lee, S.; Jung. S. Bull. Konam Chem. Soc. 2003. 24,95

11. Junn. E.: Jeong. K.: Lee. S.: Kim. T.-I.: Jung. S. Bull. Konean Chem. Soc. 2003, 24(11). 1627.

12. Syed. M. A.: Fahmeena. A.: Mannour. K. Bull. Korean Chem. Soc. 2006. 27(9). 1397. 